\title{
ODD LAPLACIANS: GEOMETRICAL MEANING OF POTENTIAL, AND MODULAR CLASS
}

\author{
H. M. KHUDAVERDIAN AND M. PEDDIE
}

\begin{abstract}
A second order self-adjoint operator $\Delta=S \partial^{2}+U$ is uniquely defined by its principal symbol $S$ and potential $U$ if it acts on half-densities. We analyse the potential $U$ as a compensating field (gauge field) in the sense that it compensates the action of coordinate transformations on the second derivatives in the same way as an affine connection compensates the action of coordinate transformations on first derivatives in the first order operator, a covariant derivative, $\nabla=\partial+\Gamma$. Usually a potential $U$ is derived from other geometrical constructions such as a volume form, an affine connection, or a Riemannian structure, etc. The story is different if $\Delta$ is an odd operator on a supermanifold. In this case the second order potential becomes a primary object. For example, in the case of an odd symplectic supermanifold, the compensating field of the canonical odd Laplacian depends only on this symplectic structure, and can be expressed by the formula obtained by K.Bering. We also study modular classes of odd Poisson manifolds via $\Delta$-operators, and consider an example of a non-trivial modular class which is related with the Nijenhuis bracket.
\end{abstract}

\section{SECOND ORDER SELF-ADJOINT OPERATOR ON HALF-DENSITIES}

Let $\mathbf{S}=S^{a b} \partial_{b} \otimes \partial_{a}$ be a rank 2, contravariant symmetric tensor field on a (super)manifold $M$. We consider the class $\mathcal{F}_{\mathbf{S}}$ of second order operators acting on half-densities on $M$ such that every operator $\Delta$ of this class obeys the following conditions:

- $\Delta$ has principal symbol $\mathbf{S}$; in local coordinates $\Delta=\frac{1}{2} S^{a b}(x) \partial_{b} \partial_{a}+\ldots$;

- Operator $\Delta$ is self-adjoint: $\Delta^{*}=\Delta$, i.e.

$$
\left\langle\Delta s_{1}, s_{2}\right\rangle=\left\langle s_{1}, \Delta^{*} s_{2}\right\rangle,
$$

where $\langle$,$\rangle is the natural scalar product on half-densities:$

$$
\left\langle\boldsymbol{s}_{1}, \boldsymbol{s}_{2}\right\rangle=\int_{M} s_{1}(x) s_{2}(x)|D x|,
$$

$\boldsymbol{s}_{1}=s_{1}(x) \sqrt{|D x|}, \boldsymbol{s}_{2}=s_{2}(x) \sqrt{|D x|}$ are two arbitrary half-densities (with compact support).

Remark 1. A density of weight $\lambda, s=s(x)|D x|^{\lambda}$, is multiplied by the $\lambda^{t h}$ power of the Jacobian under a change of coordinates. Densities of weight $\lambda=0$ are functions on the manifold, and those of weight $\lambda=1$ are volume forms. In this article we mostly consider densities of weight $\lambda=\frac{1}{2}$, half-densities.

2000 Mathematics Subject Classification. 53D17, 58A50, 81R99.

Key words and phrases. operators on half-densities, odd Poisson manifold, modular class, odd symplectic manifold, canonical odd Laplacian, compensating field. 
Proposition 1. For an arbitrary rank 2, contravariant symmetric tensor field $\mathbf{S}=\mathbf{S}(x)$ on a manifold $M$, the class $\mathcal{F}_{\mathbf{S}}$ is not empty, and any two operators in this class differ by a scalar function: if $\Delta, \Delta^{\prime} \in \mathcal{F}_{\mathbf{S}}$ then

$$
\Delta^{\prime}-\Delta=F(x)
$$

One can say that $\mathcal{F}_{\mathbf{S}}$ is an affine space of second order operators associated with the vector space of functions on $M$.

Proof. First prove (2). Indeed, operator $\Delta^{\prime}-\Delta$ has to be an operator of order $\leqslant 1$ since both operators $\Delta$ and $\Delta^{\prime}$ have the same principal symbol. The self-adjointness condition implies that the order of this operator is not equal to 1 ; if $\Delta^{\prime}-\Delta=L^{a}(x) \partial_{a}+\ldots$ then self-adjointness implies

$$
L^{a}(x) \partial_{a}+\cdots=\left(L^{a}(x) \partial_{a}+\ldots\right)^{*}=-L^{a}(x) \partial_{a}+\cdots \Rightarrow L^{a} \equiv 0 .
$$

Thus we come to equation (2).

To prove that the class $\mathcal{F}_{\mathbf{S}}$ is not empty consider (using a partition of unity argument,) an arbitrary volume form $\boldsymbol{\rho}=\rho(x)|D x|$. Now using this volume form we construct a second order operator belonging to the class $\mathcal{F}_{\mathbf{S}}$. Every half-density $\boldsymbol{s}=s(x) \sqrt{|D x|}$ defines a function $f_{s}=\frac{s}{\sqrt{\rho}}=\frac{s(x)}{\sqrt{\rho(x)}}$. This function defines a covector field $d f_{s}$, and this covector field defines a vector field $\mathbf{X}_{s}=\mathbf{S} d f_{s}$ via the contravariant tensor field $\mathbf{S}$. Considering the divergence of this vector field with respect to the volume form $\boldsymbol{\rho}$, we come to the operator $\Delta^{(\rho)}$ on half-densities defined in the following way:

$$
\begin{gathered}
\Delta^{(\boldsymbol{\rho})} \boldsymbol{s}=\frac{1}{2} \sqrt{\boldsymbol{\rho}} \operatorname{div}{ }_{\boldsymbol{\rho}} \mathbf{X}_{\boldsymbol{s}}=\frac{1}{2} \sqrt{\boldsymbol{\rho}} \operatorname{div} \boldsymbol{\rho}\left(\mathbf{S} d f_{\boldsymbol{s}}\right)=\frac{1}{2} \sqrt{\rho}(x) \frac{1}{\rho(x)} \partial_{a}\left(\rho(x) S^{a b}(x) \partial_{b}\left(\frac{s(x)}{\sqrt{\rho}(x)}\right)\right) \sqrt{|D x|} \\
=\frac{1}{2}\left(\partial_{a}\left(S^{a b} \partial_{b} s\right)-\frac{1}{2} \partial_{a}\left(S^{a b} \partial_{b} \log \rho\right) s-\frac{1}{4} \partial_{a} \log \rho S^{a b} \partial_{b} \log \rho s\right) \sqrt{|D x|}
\end{gathered}
$$

It is easy to check that it is a self-adjoint operator. Every operator $\Delta \in \mathcal{F}_{\mathbf{S}}$ differs from $\Delta^{(\rho)}$ by a scalar function: $\mathcal{F}_{\mathbf{S}} \ni \Delta=\Delta^{(\rho)}+F(x)$.

We see that self-adjointness uniquely defines a second order operator on half densities by its symbol up to a function. It is useful to look at the analogous statement for first order operators.

Proposition 2. Let $L$ be first order anti-self-adjoint operator on half-densities: $L^{*}=-L$ with principal symbol, vector field $\mathbf{X}=X^{a} \partial_{a}$. Then operator $L$ is the Lie derivative of half-densities along the field $\mathbf{X}, L=\mathcal{L}_{\mathbf{X}}$,

$$
\mathcal{L}_{\mathbf{X}}: \quad \mathcal{L}_{\mathbf{X}} s=\mathcal{L}_{\mathbf{X}}(s(x) \sqrt{|D x|})=\left(X^{a}(x) \partial_{a} s(x)+\frac{1}{2} \partial_{a} X^{a}(x) s(x)\right) \sqrt{|D x|} .
$$

Proof. Let $L=X^{a} \partial_{a}+B$. Condition of anti-self-adjointness means that

$$
L^{*}=\left(X^{a} \partial_{a}+B\right)^{*}=-X^{a} \partial_{a}-\partial_{a} X^{a}+B=-\left(X^{a} \partial_{a}+B\right) \Rightarrow B=\frac{1}{2} \partial_{a} X^{a}, \text { and } L=\mathcal{L}_{\mathbf{X}} .
$$


Corollary 1. Let $\Delta$ be an arbitrary self-adjoint second order operator on half-densities with principal symbol $\mathbf{S}=S^{a b} \partial_{b} \otimes \partial_{a}, \Delta \in \mathcal{F}_{\mathbf{S}}$. Then for an arbitrary function $f$,

$$
\Delta(f \boldsymbol{s})=f \Delta \boldsymbol{s}+\mathcal{L}_{D_{f}}, \quad \text { where } D_{f}=\mathbf{S} d f=S^{a b} \partial_{b} f \partial_{a} .
$$

Proof. The operator $[\Delta, f]=\Delta \circ f-f \circ \Delta$ is a first order operator with principal symbol, vector field $\mathbf{S} d f$. It is an anti-self-adjoint operator: $[\Delta, f]^{*}=-[\Delta, f]$. Proposition 2 then implies that equation (5) is obeyed.

Remark 2. We proved that the class $\mathcal{F}_{\mathbf{S}}$ is not empty by constructing an operator in this class via a volume form. For further considerations, it is useful to present a construction where one can define an operator in the class $\mathcal{F}_{\mathbf{S}}$ without using a volume form. To do this we note that for any principal symbol $\mathbf{S}(x)$ one can consider a finite set of vector fields $\left\{\mathbf{X}_{\lambda}, \mathbf{Y}_{\lambda}\right\}$ such that $\mathbf{S}$ can be decomposed as

$$
\mathbf{S}(x)=\sum_{\lambda} \mathbf{X}_{\lambda}(x) \otimes_{\mathrm{sym}} \mathbf{Y}_{\lambda}(x), \quad S^{a b}(x)=\frac{1}{2} \sum_{\lambda}\left(X_{\lambda}^{a}(x) Y_{\lambda}^{b}(x)+Y_{\lambda}^{a}(x) X_{\lambda}^{b}(x)\right) .
$$

Then consider an operator

$$
\Delta_{\mathbf{S}}=\Delta=\frac{1}{2} \sum_{\lambda}\left(\mathcal{L}_{\mathbf{X}_{\lambda}} \mathcal{L}_{\mathbf{Y}_{\lambda}}+\mathcal{L}_{\mathbf{Y}_{\lambda}} \mathcal{L}_{\mathbf{X}_{\lambda}}\right),
$$

where $\mathcal{L}_{\mathbf{X}}$ is the Lie derivative (4) of half-densities along the vector field $\mathbf{X}$. The principal symbol of this operator is equal to $\mathbf{S}(x)$. Due to the anti-self-adjointness of the Lie derivative of half-densities, this operator is self-adjoint. Hence equation (6) defines a self-adjoint operator on half-densities with principal symbol $\mathbf{S}$.

The relation between $\mathbf{S}$ and the set $\left\{\mathbf{X}_{\lambda}, \mathbf{Y}_{\lambda}\right\}$ of vector fields is not canonical, Considering different decompositions we come to different operators in the class $\mathcal{F}_{\mathbf{S}}$.

One can see that an arbitrary $\Delta \in \mathcal{F}_{\mathbf{S}}$ has the following appearance in local coordinates,

$$
\Delta=\frac{1}{2}\left(S^{a b}(x) \partial_{b} \partial_{a}+\partial_{b} S^{b a}(x) \partial_{a}+U(x)\right) .
$$

Definition 1. We say that $U(x)$ is a second order compensating field or just a compensating field of the second order operator $\Delta \in \mathcal{F}_{\mathbf{S}}$.

For example, for the operator (31) the compensating field is equal to

$$
U^{(\boldsymbol{\rho})}(x)=-\frac{1}{2} \partial_{a}\left(S^{a b}(x) \partial_{b} \log \rho(x)\right)-\frac{1}{4} \partial_{a} \log \rho(x) S^{a b}(x) \partial_{b} \log \rho(x) .
$$

Let us see how the compensating field transforms under a change of local coordinates $\left(x^{a}\right) \mapsto\left(x^{a^{\prime}}\right)$. The expression for a half-density in new coordinates is $s=s^{\prime}\left(x^{\prime}\right) \sqrt{\left|D x^{\prime}\right|}=$ $s^{\prime}\left(x^{\prime}(x)\right) \sqrt{J_{\left\{x^{\prime}, x\right\}}} \sqrt{|D x|}$, thus $s(x)=s^{\prime}\left(x^{\prime}(x)\right) \sqrt{J_{\left\{x^{\prime}, x\right\}}}$, where we denote

$$
J_{\left\{x^{\prime}, x\right\}}=\operatorname{det}\left(\frac{\partial x^{\prime}}{\partial x}\right) \text {. }
$$

Performing straightforward calculations, we see that in the new coordinates $\left(x^{a^{\prime}}\right)$,

$$
\begin{gathered}
\Delta s=\left(\partial_{b}\left(S^{b a}(x) \partial_{a}\left(s^{\prime}\left(x^{\prime}\right) \sqrt{J_{\left\{x^{\prime}, x\right\}}}\right)\right)+U(x) s^{\prime}\left(x^{\prime}\right) \sqrt{J_{\left\{x^{\prime}, x\right\}}}\right) \sqrt{J_{\left\{x, x^{\prime}\right\}}} \sqrt{\left|D x^{\prime}\right|}= \\
\left(\partial_{b^{\prime}}\left(S^{b^{\prime} a^{\prime}}\left(x^{\prime}\right) \partial_{a^{\prime}} s^{\prime}\left(x^{\prime}\right)\right)+U^{\prime}\left(x^{\prime}\right) s^{\prime}\left(x^{\prime}\right)\right) \sqrt{\left|D x^{\prime}\right|},
\end{gathered}
$$


where for tensor field $\mathbf{S}=S^{a b} \partial_{b} \otimes \partial_{a}$,

$$
S^{a^{\prime} b^{\prime}}\left(x^{\prime}\right)=\frac{\partial x^{a^{\prime}}}{\partial x^{a}} S^{a b}(x) \frac{\partial x^{b^{\prime}}}{\partial x^{b}}
$$

and

$$
U^{\prime}\left(x^{\prime}\right)=U(x)+\frac{1}{2} \partial_{a^{\prime}}\left(S^{a^{\prime} b^{\prime}} \partial_{b^{\prime}} \log J\right)-\frac{1}{4} \partial_{a^{\prime}} \log J S^{a^{\prime} b^{\prime}} \partial_{b^{\prime}} \log J,
$$

where $J=J_{\left\{x^{\prime}, x\right\}}$ is the Jacobian defined by equation (9).

Remark 3. In these calculations we use the fact that for Jacobian $J=J_{\left\{x^{\prime}, x\right\}}$,

$$
\frac{\partial}{\partial x^{r}} \log J=\frac{\partial x^{c}}{\partial x^{a^{\prime}}} \frac{\partial^{2} x^{a^{\prime}}}{\partial x^{c} \partial x^{r}},
$$

since for an arbitrary matrix-valued function $M, \delta \log M=\operatorname{Tr}\left(M^{-1} \delta M\right)$.

Proposition 3. An arbitrary, self-adjoint second order differential operator on half-densities is well-defined by two geometrical objects:

- The contravariant rank 2 symmetric tensor field $\mathbf{S}$ which is the principal symbol of the operator;

- A second order compensating field $U(x)$, a geometrical object which transforms under a change of coordinates according to equation (10).

For a given tensor field $\mathbf{S}$, a space of compensating fields $U$ is an affine space associated with the vector space of functions on $M$.

Consider examples of second order compensating fields.

Example 1.1. If a manifold $M$ is provided with a volume form $\boldsymbol{\rho}=\rho(x)|D x|$ then one can consider the second order compensating field $U=U^{(\boldsymbol{\rho})}$ (see equations (3) and (8)). Volume form $\boldsymbol{\rho}$ can be derived from a Riemannian metric $G(\boldsymbol{\rho}=\sqrt{\operatorname{det} G}|D x|)$, or a symplectic structure $(\boldsymbol{\rho}=|D x|$ in Darboux coordinates). See also further examples.

Example 1.2. A compensating field $U$ can be derived from a connection $\nabla$ on densities. If $\boldsymbol{s}=s(x)|D x|^{\lambda}$ is a density of weight $\lambda$ and $\mathbf{X}$ is a vector field, then the covariant derivative of density $s$ along vector field $\mathrm{X}$ is equal to

$$
\nabla_{\mathbf{X}} \boldsymbol{s}=\nabla_{X^{a} \partial_{a}}\left(s(x)|D x|^{\lambda}\right)=X^{a}\left(\partial_{a} s(x)+\lambda \gamma_{a} s(x)\right)|D x|^{\lambda},
$$

where $\left(\gamma_{a}\right)$ are such that for coordinate volume form $|D x|, \nabla_{\partial_{a}}|D x|=\gamma_{a}|D x|$. Under a change of local coordinates $\gamma_{a}$ transforms in the following way:

$$
\gamma_{a}=x_{a}^{a^{\prime}}\left(\gamma_{a^{\prime}}+\partial_{a^{\prime}} \log \operatorname{det}\left(\frac{\partial x}{\partial x^{\prime}}\right)\right)=x_{a}^{a^{\prime}} \gamma_{a^{\prime}}-x_{b^{\prime}}^{b} b_{b a}^{b^{\prime}}
$$

We use short notations for derivatives: $x_{a}^{a^{\prime}}=\frac{\partial x^{a^{\prime}}(x)}{\partial x^{a}}, x_{b c}^{a^{\prime}}=\frac{\partial x^{a^{\prime}}(x)}{\partial x^{b} \partial x^{c}}$. Symbols $\left(\gamma_{a}\right)$ can be considered as first order compensating fields.

Notice that every volume form $\boldsymbol{\rho}=\rho(x)|D x|$ induces a connection $\nabla^{(\boldsymbol{\rho})}$ on densities:

$$
\nabla_{\mathbf{X}}^{(\boldsymbol{\rho})}(\boldsymbol{s})=\boldsymbol{\rho}^{\lambda} \partial_{\mathbf{X}}\left(\boldsymbol{\rho}^{-\lambda} \boldsymbol{s}\right)=X^{a}\left(\partial_{a} s(x)+\lambda \gamma_{a}^{(\boldsymbol{\rho})}\right)|D x|^{\lambda}, \quad \gamma_{a}^{(\boldsymbol{\rho})}=-\partial_{a} \log \rho(x) .
$$


This is a flat connection. One can see that the potential $U=U^{(\boldsymbol{\rho})}$ corresponding to volume form $\boldsymbol{\rho}$ (see equation (8) and the previous example) can be expressed via the connection $\nabla^{(\boldsymbol{\rho})}$ in the following way: if $\gamma_{a}=\gamma_{a}^{(\boldsymbol{\rho})}$ then

$$
U^{(\boldsymbol{\rho})}=\frac{1}{2} \partial_{a} \gamma^{a}-\frac{1}{4} \gamma_{a} \gamma^{a}, \quad \text { where } \gamma^{a}=S^{a b} \gamma_{b}
$$

This formula works not only for a flat connection $\nabla^{(\boldsymbol{\rho})}$ defined by a volume form, but for an arbitrary connection $\nabla$ on densities. (It can be viewed in a more general framework if we consider an upper connection $\gamma^{a}$.)

A connection $\nabla$ on densities can be naturally produced via an affine connection: $\gamma_{a}=$ $-\Gamma_{b a}^{b}$, where $\Gamma_{b c}^{a}$ are the Christoffel symbols of the affine connection. If the affine connection is the Levi-Civita connection of a Riemannian manifold $(M, G)$, then it produces a flat connection corresponding to the canonical volume form of the Riemannian manifold:

$$
\gamma_{a}=-\Gamma_{b a}^{b}=-\partial_{a} \log \sqrt{\operatorname{det} G}=\gamma_{a}^{(\boldsymbol{\rho})}, \text { where } \boldsymbol{\rho}=\sqrt{\operatorname{det} G}|D x| .
$$

(See for detail [12], [13].)

In all previous examples the second order compensating field arises as a secondary object from some structure, such as a metric, volume form or first order connection. In the next example we will consider a compensating field (a potential) which arises as a primary object.

Example 1.3. For an arbitrary manifold $M$ consider its tangent bundle $T M$, and the supermanifold $\Pi T M$, where $\Pi$ is the parity reversing functor in fibres. For local coordinates $\left(x^{i}\right)$ on $M$ one can assign coordinates $\left(x^{i}, d x^{j}\right)$ on $\Pi T M$, where $\left(d x^{i}\right)$ are odd coordinates, $p\left(d x^{i}\right)=1$. Functions $F(x, d x)$ on the supermanifold $\Pi T M$ can be identified with differential forms on $M$.

One can consider on ПTM the canonical volume form $\boldsymbol{\rho}$ which, in coordinates $\left(x^{i}, d x^{j}\right)$, is equal to the coordinate volume form

$$
\boldsymbol{\rho}=|D(x, d x)| .
$$

This form remains invariant under a change of coordinates, since the Berezinian (superdeterminant) of the coordinate transformation $\left(x^{i}, d x^{j}\right) \mapsto\left(x^{i^{\prime}}, d x^{j^{\prime}}\right)$ is equal to 1 : $x^{i^{\prime}}=$ $x^{i^{\prime}}(x), d x^{j^{\prime}}=d x^{j} \frac{\partial x^{j^{\prime}}}{\partial x^{j}}$ then

$$
D\left(x^{\prime}, d x^{\prime}\right)=D(x, d x) \operatorname{Ber}\left(\frac{\partial\left(x^{\prime}, d x^{\prime}\right)}{\partial(x, d x)}\right)=D(x, d x) \operatorname{Ber}\left(\begin{array}{cc}
\frac{\partial x^{i^{\prime}}}{\partial x^{i}} & 0 \\
d x^{j} \frac{\partial^{2} x^{i}}{\partial x^{i} \partial x^{j}} & \frac{\partial x^{i^{\prime}}}{\partial x^{i}}
\end{array}\right)=D(x, d x) .
$$

We see from equations (12) and (8) that for every principal symbol $\mathbf{S}$ on $\Pi$ (1) consider a canonical potential which vanishes in coordinates $\left(x^{i}, d x^{j}\right)$.

The constructions of this example are valid if $M$ is not only a usual manifold, but an arbitrary supermanifold. In this case the coordinates $\left(x^{i}\right)$ are even and odd coordinates on $M$, and the parities of coordinates $\left(d x^{i}\right)$ are opposite to those of the coordinates $\left(x^{i}\right)$ on $M$, $p\left(d x^{i}\right)=p\left(x^{i}\right)+1$. When there are odd variables present, functions on ПTM correspond to so called pseudodifferential forms.

Remark 4. Projective connection and compensating field.

In this article we consider only operators of weight 0 , i.e. operators which do not change the weight of densities. One can consider second order operators of weight $\delta \neq 0$. A 
contravariant symmetric tensor density $\mathbf{S}|D x|^{\delta}=S^{a b} \partial_{a} \otimes \partial_{b}|D x|^{\delta}$ defines the class $\mathcal{F}_{\mathbf{S}}^{(\delta)}$ of self-adjoint second order operators of weight $\delta$ which act on densities of the weight $\frac{1-\delta}{2}$ and takes values in densities of weight $\frac{1-\delta}{2}+\delta=\frac{1+\delta}{2}$. (See for detail [12] and [13].)

In particular for the 1-dimensional case we come to the following very important construction. On the line $\mathbb{R}$, a canonical symmetric tensor density of weight $\delta=2, \partial_{x} \otimes \partial_{x}|D x|^{2}$ defines a second-order self-adjoint operator on densities of weight $\frac{1-\delta}{2}=-\frac{1}{2}$

$$
\Delta=\left(\partial_{x}^{2}+U(x)\right)|D x|^{2} .
$$

The compensating field $U(x)|D x|^{2}$ is called a projective connection. Under a change of coordinates $y=y(x)$ it transforms by the Schwartzian of the coordinate transformation:

$$
U^{\prime}(y)|D y|^{2}=U(x)|D x|^{2}-\frac{1}{2}\left(\frac{y_{x x x}}{y_{x}}-\frac{3}{2} \frac{y_{x x}^{2}}{y_{x}^{2}}\right)|D x|^{2} .
$$

(See for example [7], [16] and [13].)

\section{Second order operators on odd Poisson supermanifolds.}

The considerations of the previous section can be easily generalised for operators acting on supermanifolds, this simply involves inserting the relevant signs and using the super analogues for integrals, in particular the determinant will change to the Berezinian (superdeterminant) (as in example (1.3) ).

Most of the constructions in this section become meaningful only in the super-setting. So we will be careful about the signs arising during the calculations. For example, the definition (11) of an adjoint operator has to be rewritten $\left\langle\Delta \boldsymbol{s}_{1}, \boldsymbol{s}_{2}\right\rangle=(-1)^{p(\Delta) p\left(\boldsymbol{s}_{1}\right)}\left\langle\boldsymbol{s}_{1}, \Delta^{*} \boldsymbol{s}_{2}\right\rangle$, where $p(\Delta), p\left(\boldsymbol{s}_{1}\right)$ are the parities of the operator $\Delta$ and $\boldsymbol{s}_{1}$. For references on supermathematics see the book of F.A.Berezin [4].

Let $M$ be a supermanifold provided with an odd rank 2 contravariant symmetric tensor field $\mathbf{E}=E^{a b}(x) \partial_{b} \otimes \partial_{a}$. If $\left(x^{a}\right)$ are local coordinates on the manifold $M$ (hence forward we will usually suppress the prefix super,) then

$$
\mathbf{E}: \quad p\left(E^{a b}(x)\right)=1+p(a)+p(b), \quad E^{a b}(x)=(-1)^{p(a) p(b)} E^{b a}(x),
$$

where $p(a)$ is the parity of the coordinate $x^{a}$. We will reserve the notation $\mathbf{E}=E^{a b} \partial_{b} \otimes \partial_{a}$ for such an odd rank 2 tensor field.

Consider an arbitrary self-adjoint second order odd operator on half-densities with principal symbol E,

$$
\Delta \in \mathcal{F}_{\mathbf{E}}, \quad \Delta=\frac{1}{2} E^{a b}(x) \partial_{b} \partial_{a}+\ldots, \quad p(\Delta \boldsymbol{s})=1+p(\boldsymbol{s}) .
$$

One can see that in local coordinates it has the appearance

$$
\Delta=\frac{1}{2}\left(E^{a b} \partial_{b} \partial_{a}+\partial_{b} E^{b a} \partial_{a}+U(x)\right)
$$

where second order compensating field $U(x)$ is an odd valued function.

Consider the 'classical limit' of operator $\Delta$.

Let $\left(\right.$, ) be the canonical even Poisson bracket on the cotangent bundle $T^{*} M$ :

$$
(F, G)=(-1)^{p(a)(p(F)+1)}\left(\frac{\partial F}{\partial p_{a}} \frac{\partial G}{\partial x^{a}}-(-1)^{p(a)} \frac{\partial F}{\partial x^{a}} \frac{\partial G}{\partial p_{a}}\right),
$$


where $\left(x^{a}, p_{b}\right)$ are coordinates on $T^{*} M$ adjusted to local coordinates $\left(x^{a}\right)$ (under a change of local coordinates $\left.\left(x^{a}\right) \mapsto\left(x^{a^{\prime}}\right), p_{b^{\prime}}=\frac{\partial x^{b}}{\partial x^{b^{\prime}}} p_{b}\right)$. Then the principal symbol $\mathbf{E}$ defines on functions on the manifold $M$ the following odd bracket

$$
[F, G]=(-1)^{p(F)}\left(\left(H_{E}, F\right), G\right)=(-1)^{p(a) p(F)} \partial_{a} F E^{a b} \partial_{b} G, \quad\left[x^{a}, x^{b}\right]=(-1)^{p(a)} E^{a b},
$$

where $H_{E}=\frac{1}{2} E^{a b} p_{b} p_{a}$ is an odd Hamiltonian on $T^{*} M$, quadratic on the fibres of $T^{*} M$ (see for detail [11]). This bracket is antisymmetric with respect to a shifted parity:

$$
[F, G]=-(-1)^{(p(F)+1)(p(G)+1)}[G, F] .
$$

We say that $(M, \mathbf{E})$ is an odd Poisson manifold if $\mathbf{E}$ defines an odd Poisson bracket on $M$. That is, if the Jacobi identity for the bracket [, ] (17) is obeyed

$(-1)^{p(F) p(H)+p(G)}[F,[G, H]]+(-1)^{p(F) p(G)+p(H)}[G,[H, F]]+(-1)^{p(G) p(H)+p(F)}[H,[F, G]]=0$,

in local coordinates

$$
(-1)^{p(a)(p(c)+1)} E^{a p} \partial_{p} E^{b c}+\text { cyclic permutations }=0 .
$$

The left hand side of this equation is nothing but the canonical bracket of the odd Hamiltonian $H_{\mathbf{E}}$ with itself. Jacobi identities (18) are obeyed if and only if $\left(H_{E}, H_{E}\right)=0$. (See for detail [11].)

The fact that the tensor field $\mathbf{E}$ equips $M$ with the structure of an odd Poisson manifold can be clearly viewed in terms of operator $\Delta$.

Proposition 4. Let $\mathbf{E}=E^{a b} \partial_{b} \otimes \partial_{a}$ be an odd contravariant symmetric tensor field on a supermanifold $M$, and $\Delta$ be an arbitrary second order odd self-adjoint operator with principal symbol $\mathbf{E}, \Delta=E^{a b} \partial_{b} \partial_{a}+\ldots,\left(\Delta \in \mathcal{F}_{\mathbf{E}}(M)\right)$. Then

- Operator $\Delta^{2}=\frac{1}{2}[\Delta, \Delta]$ is an even anti-self-adjoint operator, $\left(\Delta^{2}\right)^{*}=-\Delta^{2}$, which has order equal to 3,1 , or else $\Delta^{2}=0$.

- Operator $\Delta^{2}$ is of order 1 , or else $\Delta^{2}=0$, if and only if $\mathbf{E}$ defines an odd Poisson structure on $M$, i.e. if $(M, \mathbf{E})$ is an odd Poisson supermanifold.

- If $(M, \mathbf{E})$ is an odd Poisson supermanifold then an even anti-self-adjoint operator $\Delta^{2}$ is equal to the Lie derivative along some vector field $\mathbf{X}$ :

$$
\Delta^{2}=\mathcal{L}_{\mathbf{X}}
$$

This equation assigns an even vector field $\mathbf{X}=\mathbf{X}(\Delta)$ to the operator $\Delta$ defining the odd Poisson structure. We call the vector field $\mathbf{X}$ the modular vector field of the operator $\Delta$. The modular vector field $\mathbf{X}(\Delta)$ is a Poisson vector field, that is, it preserves the odd Poisson structure.

- If $\Delta^{\prime}=\Delta+F$ is another odd operator defining the same Poisson structure $\left(\Delta, \Delta^{\prime} \in\right.$ $\mathcal{F}_{\mathbf{E}}(M)$, ) then

$$
\mathbf{X}\left(\Delta^{\prime}\right)=\mathbf{X}(\Delta)+D_{F},
$$

where $D_{F}$ is the even Hamiltonian vector field corresponding to the odd function $F$ : $D_{F} G=[F, G]$ for an arbitrary function $G$.

Proof. The first statement of the Proposition follows from the fact that for two arbitrary operators $\Delta_{1}, \Delta_{2}$

$$
\left[\Delta_{1}, \Delta_{2}\right]^{*}=\left[\Delta_{1} \circ \Delta_{2}-(-1)^{p\left(\Delta_{1}\right) p\left(\Delta_{2}\right)} \Delta_{2} \circ \Delta_{1}\right]^{*}=(-1)^{p\left(\Delta_{1}\right) p\left(\Delta_{2}\right)} \Delta_{2}^{*} \circ \Delta_{1}^{*}-\Delta_{1}^{*} \circ \Delta_{2}^{*}=-\left[\Delta_{1}^{*}, \Delta_{2}^{*}\right] \text {. }
$$


Thus for self-adjoint operators their commutator is anti-self-adjoint. Since $\Delta$ is odd, $\Delta^{2}=$ $\frac{1}{2}[\Delta, \Delta]$ is a commutator operator, so its order is less than or equal to 3 . For the even operator $\Delta^{2}$,

$$
\Delta^{2}=P^{a b c} \partial_{c} \partial_{b} \partial_{a}+\cdots=\left(E^{a b} \partial_{b} \partial_{a}+\ldots\right)^{2}=2(-1)^{b} E^{b a} \partial_{a} E^{c d} \partial_{d} \partial_{c} \partial_{b}+\ldots
$$

The principal symbol of $\Delta^{2}$ defines a cubic Hamiltonian $\left(H_{\mathbf{E}}, H_{\mathbf{E}}\right)=2(-1)^{b} E^{b a} \partial_{a} E^{c d} p_{d} p_{c} p_{b}$. The vanishing of this cubic Hamiltonian is equivalent to the Jacobi identity (18) for the odd bracket (17). Therefore the Jacobi identity is obeyed if and only if the order of $\Delta^{2}$ is less than 3. One can say more. The order of an anti-self-adjoint operator $\Delta^{2}$ cannot be equal to 2 or 0 . Indeed, if $\Delta^{2}=L^{a b} \partial_{b} \partial_{a}+P^{a} \partial_{a}+F$ then $\left(\Delta^{2}\right)^{*}=-\Delta^{2}=-L^{a b} \partial_{b} \partial_{a}+\ldots$, hence $L^{a b} \equiv 0$, i.e. the order of operator $\Delta^{2}$ is $\leqslant 1$. If the $P^{a}$ also vanish, i.e. the order of $\Delta^{2}$ is $<1$, then anti-self-adjointness implies that $F$ also vanishes, i.e. $\Delta^{2}=0$. We see that the order of the operator $\Delta^{2}$ can be equal to either 3,1 , or $\Delta^{2}=0$. Therefore if $\mathbf{E}$ defines an odd Poisson structure on $M$ then $\Delta^{2}$ is an even first order operator (or it vanishes). This operator is an anti-self-adjoint, even operator. Hence it is equal to the Lie derivative $\mathcal{L}_{\mathbf{X}}$ along a vector field $\mathbf{X}$, which is its principal symbol (see Proposition [2): $\Delta^{2}=\mathcal{L}_{\mathbf{X}}=X^{a} \partial_{a}+\frac{1}{2}(-1)^{a} \partial_{a} X^{a}$.

The vector field $\mathbf{X}$ preserves the operator $\Delta: \mathcal{L}_{\mathbf{X}} \circ \Delta=\Delta^{2} \circ \Delta=\Delta \circ \Delta^{2}=\Delta \circ \mathcal{L}_{\mathbf{X}}$. Hence it preserves the odd Poisson bracket (17), defined by the principal symbol of this operator. The proof of equation (20) follows from Corollary 1 . Since $F$ is an odd function then

$$
\left(\Delta^{\prime}\right)^{2}=(\Delta+F)^{2}=\Delta^{2}+[\Delta, F]=\mathcal{L}_{\mathbf{X}}+\mathcal{L}_{\mathbf{E} d f}=\mathcal{L}_{\mathbf{X}+D_{F}}
$$

Hamiltonian vector fields evidently preserve the Poisson bracket, they are Poisson vector fields. Consider the first Lichnerowicz-Poisson cohomology group of the Poisson manifold $(M, \mathbf{E})$

Using the Proposition above we come to

$$
H_{L P}^{1}(M, \mathbf{E})=\frac{\text { Poisson vector fields on } M}{\text { Hamiltonian vector fields on } M} .
$$

Definition 2. Let $(M, \mathbf{E})$ be a supermanifold provided with an odd Poisson structure, and let $\Delta$ be an arbitrary odd second order self-adjoint operator on half-densities, such that $\mathbf{E}$ is its principal symbol, $\Delta \in \mathcal{F}_{\mathbf{E}}(M)$. Let $\mathbf{X}=\mathbf{X}(\Delta)$ be the modular vector field (19) of operator $\Delta$. Due to equation (20) , the equivalence class $[\mathbf{X}]$ in $H_{L P}^{1}(M, \mathbf{E})$ does not depend on a choice of operator $\Delta$ in the class $\mathcal{F}_{\mathbf{E}}(M)$. We call this class the modular class of the odd Poisson supermanifold $(M, \mathbf{E})$.

It is useful to write down explicit formulae for the modular vector field of a self-adjoint operator $\Delta$. If $\Delta$ has the appearance (15) in local coordinates, then

$\Delta^{2}=\mathcal{L}_{\mathbf{X}}=X^{a} \partial_{a}+\frac{1}{2}(-1)^{p(b)} \partial_{b} X^{b}=\left(\frac{1}{2} \partial_{b}\left(E^{b c} \partial_{c} \partial_{p} E^{p a}\right)+(-1)^{p(a)} E^{a b} \partial_{b} U\right) \partial_{a}+\frac{1}{2} \partial_{b}\left(E^{b p} \partial_{p} U\right)$, i.e.

$$
\mathbf{X}=X^{a} \partial_{a}=\left(\frac{1}{2} \partial_{b}\left(E^{b c} \partial_{c} \partial_{p} E^{p a}\right)+(-1)^{p(a)} E^{a b} \partial_{b} U\right) \partial_{a}
$$

Let us make the following remark regarding the origin of this construction, and in particular about its counterpart for an even Poisson structure. 
Let $(M, \mathbf{P})$ be a usual manifold (without supervariables) with a Poisson structure defined by an even Poisson tensor $\mathbf{P}$. For an arbitrary volume form $\boldsymbol{\rho}=\rho(x)|D x|$ on $M$, one can consider the modular vector field $\mathbf{X}=\mathbf{X}^{(\boldsymbol{\rho})}$ such that for an arbitrary function $F$

$$
\mathbf{X}^{(\boldsymbol{\rho})}(F)=\frac{\mathcal{L}_{D_{F}} \boldsymbol{\rho}}{\boldsymbol{\rho}}=\operatorname{div}_{\boldsymbol{\rho}} D_{F}=\frac{1}{\rho(x)} \partial_{a}\left(\rho(x) P^{a b} \partial_{b} F\right)
$$

where $D_{F}$ is as usual the Hamiltonian vector field corresponding to $F: D_{F} G=\{F, G\}_{\mathbf{P}}$.

One can see that $\mathbf{X}^{(\boldsymbol{\rho})}$ is a Poisson vector field. If we choose another volume form $\boldsymbol{\rho}^{\prime}=e^{G} \boldsymbol{\rho}$, then it is easy to see that $\mathbf{X}^{(\boldsymbol{\rho})}$ changes by a Hamiltonian vector field: $\mathbf{X}^{\left(\boldsymbol{\rho}^{\prime}\right)}=\mathbf{X}^{(\boldsymbol{\rho})}+D_{G}$ (compare with equation (20) $)$. Thus one can assign to the Poisson manifold $(M, \mathbf{P})$ an equivalence class $\left[\mathbf{X}^{(\boldsymbol{\rho})}\right]$ of vector field (22) in the cohomology group $H_{L P}^{1}(M, \mathbf{P})$. It is how A.Weinstein defined the modular class of a usual Poisson manifold [19].

Weinstein's modular class vanishes for an even symplectic manifold when the Poisson tensor $\mathbf{P}$ is invertible; in this case there exists an invariant volume form $\boldsymbol{\rho}$. If $\mathcal{G}$ is a Lie algebra with structure constants $c_{k m}^{i}$, then the modular class of the Lie-Poisson bracket $\left\{u_{i}, u_{k}\right\}=c_{i k}^{m} u_{m}$ is represented by the covector $t_{m}=c_{i m}^{i}$.

The Weinstein construction of modular class can be easily performed for an even Poisson supermanifold, but remarkably, for an odd Poisson manifold equation (22) does not define a vector field. The operation which assigns to an arbitrary function $F$ the divergence of the Hamiltonian vector field $D_{F}$ with respect to an odd Poisson structure is no longer a first order differential operator. It becomes a second order operator (see detail in [8] and [9])

$$
\Delta_{\rho} F=\frac{1}{2} \frac{\mathcal{L}_{D_{F}} \boldsymbol{\rho}}{\boldsymbol{\rho}}=\frac{1}{2} \operatorname{div}_{\boldsymbol{\rho}} D_{F} .
$$

Here $\frac{1}{2}$ is just a normalisation coefficient, and $D_{F} G=[F, G]_{\mathbf{E}}$ where $[,]_{\mathbf{E}}$ is the odd bracket of this odd Poisson manifold. We change notation for operation (22) stressing the fact that for an odd Poisson structure it becomes a second order operator. The odd Poisson tensor $\mathbf{E}$ defining the odd Poisson structure is a symmetric tensor (see (14)) and it is equal to the principal symbol of this second order operator $\Delta_{\rho}$. (The Poisson tensor $\mathbf{P}$ defining the usual Poisson structure and the modular vector field $\mathbf{X}^{(\boldsymbol{\rho})}$ is an antisymmetric tensor (see detail in [8], [9] and [11])).

For example consider an odd symplectic case, a non-degenerate $n \mid n$-dimensional odd Poisson manifold. (The equality of odd and even dimensions is implied by the non-degeneracy of the odd bracket.) Let $\left(q^{i}, \theta_{j}\right), i, j=1, \ldots, n$ be even and odd Darboux coordinates of this supermanifold:

Then

$$
\left[q^{i}, \theta_{j}\right]=\delta_{j}^{i},\left[q^{i}, q^{j}\right]=0,\left[\theta_{i}, \theta_{j}\right]=0
$$

$$
\Delta_{\boldsymbol{\rho}} F=\frac{1}{2} \frac{\mathcal{L}_{D_{F}} \boldsymbol{\rho}}{\boldsymbol{\rho}}=\frac{1}{2} \operatorname{div}_{\boldsymbol{\rho}} D_{F}=\frac{\partial^{2} F(q, \theta)}{\partial q^{i} \partial \theta_{i}}+\frac{1}{2}[\log \rho, F] .
$$

In the case if $\boldsymbol{\rho}=|D(q, \theta)|$ is a coordinate volume form, then this operator becomes the famous Batalin-Vilkovisky $\Delta$-operator which was introduced in the seminal work [3]. (See [8], [17], [10].)

Operator (25) and the related operator on half-densities were studied in the article [1] for an arbitrary odd Poisson manifold. In particular, in this article it was noticed that the operator $\Delta_{\boldsymbol{\rho}}^{2}$ is a vector field and that $\Delta_{\boldsymbol{\rho}^{\prime}}^{2}=\Delta_{e^{G} \boldsymbol{\rho}}^{2}=\Delta_{\boldsymbol{\rho}}^{2}+D_{G}$ if $\boldsymbol{\rho}^{\prime}=e^{G} \boldsymbol{\rho}$. From this, the 
definition of modular class was suggested for an odd Poisson manifold. One can see that this definition of modular class in [11] is equivalent to definition (2) given here. On the other hand, the considerations in [11] were performed straightforwardly without using selfadjointness property of operators on half-densities, and examples of odd Poisson manifolds with a non-trivial modular class were not considered.

Remark 5. Notice that if $\boldsymbol{\rho}$ is an arbitrary volume form on an odd Poisson manifold $(M, \mathbf{E})$ then operator $\Delta^{(\boldsymbol{\rho})} \in \mathcal{F}_{\mathbf{E}}$ on half-densities with second order compensation field $U=U^{(\boldsymbol{\rho})}$ constructed with use of compensation field $U$ (see equations (11) and (88)), and the operator $\Delta_{\rho}$ on functions constructed in equation (25) are related by equation

$$
\Delta^{(\rho)}=\sqrt{\rho} \circ \Delta_{\rho} \circ \frac{1}{\sqrt{\rho}} .
$$

\section{Examples. Calculation of modular Classes.}

We consider here examples of second order self-adjoint odd operators which define an odd Poisson bracket. We study the odd potential of these operators, and calculate the modular classes of these Poisson manifolds.

3.1. An odd symplectic manifold. Bering's formula. Let $(M, \mathbf{E})$ be an odd symplectic manifold, i.e. an odd Poisson tensor field $\mathbf{E}$ defines a non-degenerate odd Poisson structure. The odd differential form $\mathbf{e}=d x^{a} d x^{b} e_{a b}(x)$ is inverse to the odd Poisson tensor $\mathbf{E}=E^{a b}(x) \partial_{b} \otimes \partial_{a}: E^{a c}(x) e_{c b}(x)=\delta_{b}^{a}$.

The basic example of an odd symplectic manifold is the following: for an arbitrary $n$ dimensional manifold $L$, consider its cotangent bundle reversing the parity of coordinates in the fibres, i.e. an $n \mid n$-dimensional supermanifold $M=\Pi T^{*} L$. One can assign to local coordinates $\left(q^{i}\right)$ on the manifold $L$, local coordinates $w^{a}=\left(q^{i}, \theta_{j}\right),(i, j=1, \ldots, n)$ on the supermanifold $\Pi T^{*} L$, where $\theta_{j}$ are odd coordinates in the fibres, the odd conjugate momenta (under a change of local coordinates $\left(q^{i}\right) \mapsto\left(q^{i^{\prime}}\right), \theta_{j^{\prime}}=\frac{\partial q^{j}}{\partial q^{j^{\prime}}} \theta_{j}$ ). Functions on the supermanifold $M=\Pi T^{*} L$ can be identified with multivector fields on $L$. In the same way as the cotangent bundle $T^{*} L$ possesses the usual canonical even symplectic structure, the supermanifold $\Pi T^{*} L$ possesses canonical odd symplectic structure; local coordinates $\left(q^{i}, \theta_{j}\right)$ become Darboux coordinates (see equation (24)) of this odd symplectic supermanifold. The initial manifold $L$ is a Lagrangian $n \mid 0$-dimensional surface in this symplectic space. One can show that every odd symplectic supermanifold is symplectomorphic to $\Pi T^{*} L$, where $L$ is its $n \mid 0$-dimensional Lagrangian surface (see [17] and [10]).

On the odd symplectic supermanifold $(M, \mathbf{E})$, consider an arbitrary odd second order selfadjoint operator on half-densities with principal symbol E. In arbitrary local coordinates it has the appearance given by equation (15). An odd potential $U(x)$ can be defined, for example, via a volume form or a connection on densities (see equations (8) and (11)), but in the case when $M$ is an odd symplectic supermanifold one can define an odd potential $U(x)$ as a primary object in the following way: choose instead of local coordinates $\left(x^{a}\right)$, arbitrary Darboux coordinates $w^{a}=\left(q^{i}, \theta_{j}\right)$ and define the operator $\Delta$ in these Darboux coordinates by the equation

$$
\Delta: \quad \Delta s=\Delta(s(q, \theta) \sqrt{|D(q, \theta)|})=\frac{\partial^{2} s(q, \theta)}{\partial q^{i} \partial \theta_{i}} \sqrt{|D(q, \theta)|} .
$$


The remarkable fact is that this formula gives a well-defined operator, since expression (26) does not depend on a choice of Darboux coordinates (see for detail [10]). We call this operator the canonical odd Laplacian of an odd symplectic manifold.

In Darboux coordinates, the potential of the odd canonical Laplacian vanishes. Klaus Bering calculated in [5] the expression for the odd potential $U(x)$ of the canonical odd Laplacian in arbitrary coordinates. In our notation it looks as follows:

$$
U(x)=\frac{1}{4} \partial_{b} \partial_{a} E^{a b}(x)-(-1)^{p(b)(p(d)+1)} \frac{1}{12} \partial_{a} E^{b c}(x) e_{c d}(x) \partial_{b} E^{d a}(x) .
$$

One can see by straightforward calculations that the right hand side of this expression transforms as a second order compensating field (10) under infinitesimal and linear coordinate transformations (see [5]). Alternatively one can come to this answer by the following considerations: if $\left(w^{a}\right)$ are arbitrary Darboux coordinates, and $x^{a}=x^{a}\left(w^{a^{\prime}}\right)$ then $E^{a b}(x)=(-1)^{p(a)\left(p\left(a^{\prime}\right)+1\right)} x_{a^{\prime}}^{a} a^{a^{\prime} b^{\prime}} x_{b^{\prime}}^{b}$, where $I^{a^{\prime} b^{\prime}}$ are components (they are constants) of the matrix of tensor $\mathbf{E}$ in Darboux coordinates. Using (10) we will arrive at Bering's formula. We discuss this formula later in the next section.

The modular vector field of the canonical odd Laplacian vanishes since $(\Delta)^{2}=0$. This is evident in Darboux coordinates. Hence the modular class of an odd symplectic manifold vanishes.

We see that the modular class of an odd symplectic manifold vanishes as in the even case, despite the absence of an invariant volume form (see for detail [11]).

3.2. Koszul bracket. Consider in this example another canonical construction of an odd Poisson structure, which is now not necessarily symplectic.

Let $(M, \mathbf{P})$ be an arbitrary usual Poisson manifold. The Poisson structure is defined on $M$ by a rank 2 contravariant antisymmetric tensor $\mathbf{P}$ obeying the Jacobi identity. One can assign to $(M, \mathbf{P})$ an odd Poisson supermanifold in the following way. Consider as in example1.3. the supermanifold ПTM (the tangent bundle to $M$ with parity reversed fibres). Functions on $\Pi T M$ can be identified with differential forms on the manifold $M$. It is convenient to work on $\Pi T M$ in local coordinates $\left(x^{i}, d x^{j}\right)$, where $\left(x^{i}\right)$ are coordinates on $M\left(x^{i}\right.$ are even and $d x^{i}$ odd variables). The usual Poisson bracket $\{\}=,\{,\}_{\mathbf{P}}$ on $M$ can be canonically lifted to an odd Poisson bracket (the Koszul bracket) [, ] on ПТ $M$ in the following way: for two arbitrary functions $F, G$ on $M$

$$
[F, G]=0, \quad[F, d G]=-\{F, G\}_{\mathbf{P}}, \quad[d F, d G]=-d\{F, G\}_{\mathbf{P}} .
$$

These relations give a well-defined odd bracket. In local coordinates, if $\left\{x^{i}, x^{j}\right\}=P^{i j}$, then

$$
\left[x^{i}, x^{j}\right]=0, \quad\left[x^{i}, d x^{j}\right]=\left[d x^{i}, x^{j}\right]=-P^{i j}, \quad\left[d x^{i}, d x^{j}\right]=-d P^{i j}=-d x^{k} \partial_{k} P^{i j} .
$$

Thus we define an odd Poisson structure (Koszul bracket) $\mathbf{E}_{\mathbf{P}}$ on $\Pi T M$ via the even Poisson structure on the manifold $M$. The matrix of the tensor $\mathbf{E}_{\mathbf{P}}$ in coordinates $\left(x^{i}, d x^{j}\right)$ is the following:

$$
E_{\mathbf{P}}^{a b}(x, d x)=\left(\begin{array}{cc}
0 & -P^{i j}(x) \\
P^{i j} & d x^{k} \partial_{k} P^{i j}(x)
\end{array}\right)
$$

where $\| E_{\mathbf{P}}^{a b}||$ is an $n|n \times n| n$ odd matrix, $n$ is the dimension of the Poisson manifold $M$, and $\left\|P^{i j}\right\|$ is the $n \times n$ matrix of the Poisson tensor $\mathbf{P}$. 
One can consider a canonical volume form $\boldsymbol{\rho}$ on $\Pi T M$ such that in coordinates $\left(x^{i}, d x^{j}\right)$, $\boldsymbol{\rho}=|D(x, d x)|$ (see equation (12)). Let $\Delta$ be a second order self-adjoint operator on halfdensities on the odd Poisson manifold (ПTM, $\mathbf{E}_{\mathbf{P}}$ ), $\Delta \in \mathcal{F}_{\mathbf{E}_{\mathbf{P}}}$, with a second order compensating field, an odd potential $U$, defined by this canonical volume form, $U=U^{(\boldsymbol{\rho})}, \Delta=\Delta^{(\boldsymbol{\rho})}$ (see equations (11) and (8)). In other words, the operator $\Delta$ has principal symbol $\mathbf{E}_{\mathbf{P}}$ and has the appearance of (15), where the tensor $\mathbf{E}_{\mathbf{P}}=\left\|E_{\mathbf{P}}^{a b}\right\|$ is defined by the Koszul bracket (29), and the potential $U=U^{(\boldsymbol{\rho})}$ vanishes in local coordinates $(x, d x)$.

The canonical volume form $\boldsymbol{\rho}$ on $\Pi T M$ identifies the operator $\Delta=\Delta^{(\rho)}$ on half-densities with an operator $\Delta_{\rho}$ on functions on ПTM (see the equation in remark 5), and further with the Koszul-Brylinski operator $\partial_{\mathbf{P}}$. The Koszul-Brylinski operator is the operator on differential forms which is defined by the equation $\partial_{\mathbf{P}}=\left[d, \iota_{\mathbf{P}}\right]$, where $d=d x^{a} \frac{\partial}{\partial x^{a}}$ is the de Rham differential, and $\iota_{\mathbf{P}}$ is the interior product with the bivector $\mathbf{P}$. If the volume form is canonical, the identification of differential forms on $M$ with functions on ПT $M$ identifies the two operators $\partial_{\mathbf{P}}$ and $\Delta_{\boldsymbol{\rho}}$. The Jacobi identity for the Poisson tensor $\mathbf{P}$ implies that $\boldsymbol{\iota}_{\mathbf{P}}^{2}=0$, and hence for the operator $\Delta$ on half-densities, $\Delta^{2}=0$ also. Since $\Delta^{2}=0$, the modular vector field of the operator $\Delta$ vanishes, and therefore the modular class of the odd Poisson manifold (ПTM, P) vanishes. (See [15] for details, where the relation between the Koszul-Brylinski operator and the operator $\Delta_{\rho}$ on functions was studied.)

Remark 6. The vanishing of the modular vector field for operator $\Delta$ on half-densities can be checked by the following straightforward considerations. In coordinates $\left(x^{i}, d x^{j}\right)$, the odd potential $U=U^{(\boldsymbol{\rho})}$ vanishes, and the modular vector field $\mathbf{X}$ of the operator $\Delta$ according to equation (21) has the following appearance

$$
\mathbf{X}(\Delta)=\frac{1}{2} \partial_{b}\left(E_{\mathbf{P}}^{b c}(x, d x) \partial_{c} \partial_{p} E_{\mathbf{P}}^{p a}(x, d x)\right) \partial_{a} .
$$

Notice that if a point $x$ of manifold $M$ is regular, (i.e. rank of Poisson tensor $\mathbf{P}$ is locally constant in a vicinity of this point,) then one can find in a vicinity of this point Darboux-Lie coordinates such that in these coordinates, the components $\left\|P^{i j}\right\|$ of the Poisson tensor $\mathbf{P}$ are constants (see [18 for details). This means that the entries of the matrix $\left\|E_{\mathbf{P}}^{a b}\right\|$ of the odd Poisson tensor $\mathbf{E}_{\mathbf{P}}$ (see (29)) are also constants. Hence equation (30) implies that $\mathbf{X}(\Delta)$ vanishes at regular points of $M$. Thus the modular field vanishes at all points, since regular points are dense in $M$.

3.3. Example of non-trivial modular class. Now we consider an example of an odd Poisson manifold with a non-vanishing modular class.

Let $N$ be an arbitrary $p \mid q$-dimensional supermanifold. We add to $N$ an additional coordinate -'odd time', by considering a new $p \mid q+1$-dimensional supermanifold

$$
\mathcal{N}=N \times \Pi \mathbb{R}
$$

where $\Pi \mathbb{R}$ is the $0 \mid 1$-dimensional odd line. Local coordinates on the supermanifold $\mathcal{N}$ are $\left(x^{a}, \tau\right)$, where $x^{a}$ are even and odd coordinates on $N$, and $\tau$ is an odd coordinate on $\Pi \mathbb{R}$.

The construction of the supermanifold $\mathcal{N}=N \times \Pi \mathbb{R}$ is in a certain precise sense adjoint to the construction of the supermanifold $\Pi T M$, which we used in example 1.3 and in the construction in subsection 3.2, Namely

$$
\operatorname{Maps}(N \times \Pi \mathbb{R}, M)=\operatorname{Maps}(N, \Pi T M), \quad \text { (natural isomorphism) }
$$


since

$$
\Pi T M=\underline{\operatorname{Maps}}(\Pi \mathbb{R}, M), \quad \text { (the supermanifold of 'odd paths') . }
$$

Let $\boldsymbol{\eta}$ be an arbitrary odd vector field on supermanifold $N, \boldsymbol{\eta}=\eta^{a}(x) \partial_{a},(p(\boldsymbol{\eta})=1)$. Consider on the supermanifold $\mathcal{N}=N \times \Pi \mathbb{R}$ two vector fields, even and odd, $\mathbf{A}=\tau \boldsymbol{\eta}$ and $\mathbf{B}=\frac{\partial}{\partial \tau}$. These vector fields define a second order, odd self-adjoint operator on half-densities

$$
\Delta=\frac{1}{2}\left(\mathcal{L}_{\mathbf{A}} \circ \mathcal{L}_{\mathbf{B}}+\mathcal{L}_{\mathbf{B}} \circ \mathcal{L}_{\mathbf{A}}\right)=\frac{1}{2}\left(\mathcal{L}_{\tau \boldsymbol{\eta}} \circ \mathcal{L}_{\partial_{\tau}}+\mathcal{L}_{\partial_{\tau}} \circ \mathcal{L}_{\tau \eta}\right)
$$

where $\mathcal{L}_{\mathbf{A}}, \mathcal{L}_{\mathbf{B}}$ are the Lie derivatives of half-densities. (See equation (6) in Remark2.) In local coordinates

and so

$$
\mathcal{L}_{\mathbf{A}}=\tau \eta^{a}(x) \partial_{a}+\frac{1}{2} \tau \partial \eta,\left(\partial \eta=\partial_{a} \eta^{a}(x)\right), \quad \mathcal{L}_{\mathbf{B}}=\frac{\partial}{\partial \tau},
$$

$$
\Delta=\tau \eta^{a}(x) \partial_{a} \partial_{\tau}+\frac{1}{2} \eta^{a}(x) \partial_{a}+\frac{1}{2} \tau \partial \eta \partial_{\tau}+\frac{1}{4} \partial \eta,
$$

One can see by straightforward calculations that

$$
\Delta^{2}=\frac{1}{4} \eta^{a} \partial_{a} \eta^{b} \partial_{b}+\frac{1}{8} \eta^{a} \partial_{a} \partial \eta=\mathcal{L}_{\mathbf{X}}, \quad \text { where } \mathbf{X}=\mathbf{X}(\Delta)=\frac{1}{4} \eta^{b} \partial_{b} \eta^{a} \partial_{a}=\frac{1}{4} \boldsymbol{\eta} \boldsymbol{\eta}=\frac{1}{8}[\boldsymbol{\eta}, \boldsymbol{\eta}]
$$

Since $\Delta^{2}$ is a vector field, Proposition 4 tells us that the principal symbol of the operator $\Delta$ defines an odd Poisson structure $\{$,$\} on \mathcal{N}$ given in local coordinates as

$$
\left\{x^{a}, \tau\right\}=-\left\{\tau, x^{a}\right\}=\tau \eta^{a},\left\{x^{a}, x^{b}\right\}=0,\{\tau, \tau\}=0 .
$$

Consider the modular vector field $\mathbf{X}$ defined by equation (32). One can see that if $\mathbf{X} \neq 0$, then the modular class of this Poisson manifold does not vanish. Indeed, the Poisson bracket (33) of two arbitrary functions belongs to the ideal generated by the odd variable $\tau$, it is proportional to $\tau$, the 'odd time'. Thus all the components of an arbitrary Hamiltonian vector field are proportional to $\tau$ since $D_{F}=\left\{F, x^{a}\right\} \partial_{a}+\{F, \tau\} \partial_{\tau}$. On the other hand, the components of the modular vector field (32) do not depend on $\tau$. Hence the modular class $[\mathbf{X}]$ of the modular vector field (32) is not trivial in the cohomology group $H_{L P}^{1}(\mathcal{N}, \mathbf{E})$ of the Poisson manifold $(\mathcal{N}, \mathbf{E}$ ) (if $\mathbf{X} \neq 0$ ). The considerations of this example can be summarised in the following statement.

Theorem 1. Let $N$ be an arbitrary supermanifold, and let $\boldsymbol{\eta}$ be an arbitrary odd vector field on this supermanifold. The pair $(N, \boldsymbol{\eta})$ defines the odd Poisson manifold $\mathcal{N}=N \times \Pi \mathbb{R}$ with Poisson structure expressed via the principal symbol of second order operator (31) (see equations (33) ).

The even vector field $\mathbf{X}=\frac{1}{8}[\boldsymbol{\eta}, \boldsymbol{\eta}]$ is the modular vector field of this operator $\left(\Delta^{2}=\mathcal{L}_{\mathbf{X}}\right)$, and this vector field (if it does not vanish,) defines a non-trivial modular class of this odd Poisson supermanifold.

In the next example we consider a special case of this modular class.

Remark 7. Notice that in particular $C(N \times \Pi \mathbb{R})=C(N) \oplus C(\Pi N)$. For an arbitrary function $F(x, \tau)$ on $N \times \Pi \mathbb{R}$

$$
F(x, \tau)=f(x)+\tau g(x) \mapsto\left(\begin{array}{c}
f(x) \\
g(x)
\end{array}\right) .
$$


One can see that in this representation the actions (31), (32) of operators $\Delta$ and $\Delta^{2}$ have the following appearance:

$$
\Delta\left(\begin{array}{l}
f(x) \\
g(x)
\end{array}\right)=\frac{1}{2} \mathcal{L}_{\boldsymbol{\eta}}\left(\begin{array}{c}
f(x) \\
g(x)
\end{array}\right)
$$

and

$$
\Delta^{2}\left(\begin{array}{c}
f(x) \\
g(x)
\end{array}\right)=\frac{1}{4} \mathcal{L}_{\boldsymbol{\eta}}^{2}\left(\begin{array}{c}
f(x) \\
g(x)
\end{array}\right)=\frac{1}{4} \mathcal{L}_{\boldsymbol{\eta}} \circ \mathcal{L}_{\boldsymbol{\eta}}\left(\begin{array}{c}
f(x) \\
g(x)
\end{array}\right)=\frac{1}{8} \mathcal{L}_{[\boldsymbol{\eta}, \boldsymbol{\eta}]}\left(\begin{array}{c}
f(x) \\
g(x)
\end{array}\right) .
$$

3.4. Modular class and Nijenhuis bracket. In this example, using Theorem 1, we study the relation between the Nijenhuis bracket of form-valued vector fields and modular classes.

The Nijenhuis bracket is the bracket of vector fields with values in differential forms. Recall its construction. Let $\mathbf{X}=\mathbf{X}(x, d x)$ be a vector field with values in differential forms on a manifold $M$, e.g. $\mathbf{X}=d x^{j} X_{j}^{i}(x) \partial_{i}$ is a vector field with values in 1-forms on $M$ (which corresponds to a linear operator on tangent vectors to $M$ ).

Consider the supermanifold ПTM (see examples [1.3, 3.2). Every vector field $\mathbf{X}(x, d x)$ with values in differential forms can be lifted to a vector field on the supermanifold $\Pi T M$ :

$$
\mathbf{X}=X^{i}(x, d x) \partial_{i} \mapsto \hat{\mathbf{X}}=X^{i}(x, d x) \partial_{i}+(-1)^{p(\mathbf{X})} d x^{k} \frac{\partial}{\partial x^{k}} X^{i}(x, d x) \frac{\partial}{\partial d x^{i}}
$$

The lifting is uniquely defined by the condition that the lifted field $\hat{\mathbf{X}}$ commutes with the de Rham differential $d=d x^{k} \frac{\partial}{\partial x^{k}}$ :

$$
\widehat{\mathbf{X}}: \quad\left\{\begin{array}{l}
p_{*} \widehat{\mathbf{X}}=\mathbf{X}, \quad p: \Pi T M \rightarrow M \\
{[\widehat{\mathbf{X}}, d]=0 .}
\end{array}\right.
$$

The Nijenhuis bracket $[\mathbf{X}, \mathbf{Y}]_{N}$ of form-valued vector fields $\mathbf{X}, \mathbf{Y}$ can be defined as a formvalued vector field such that

$$
[\mathbf{X}, \mathbf{Y}]_{N}: \quad\left[\widehat{\mathbf{X}, \mathbf{Y}]_{N}}=[\widehat{\mathbf{X}}, \widehat{\mathbf{Y}}]\right.
$$

The RHS of this equation is just the usual commutator of vector fields on the supermanifold $\Pi T M$ :

$$
[\widehat{\mathbf{X}}, \widehat{\mathbf{Y}}]=\widehat{\mathbf{X}} \widehat{\mathbf{Y}}-(-1)^{p(\hat{\mathbf{X}}) p(\hat{\mathbf{Y}})} \widehat{\mathbf{Y}} \widehat{\mathbf{X}}
$$

(Details of the construction above for the Nijenhuis bracket can be found e.g. in [14]. This bracket was discovered in the 1950s and predates supermathematics, however it is through the language of supermathematics that this construction is best described.)

In particular, if $\mathbf{X}(x, d x)$ is odd, i.e. it takes values in usual differential forms of rank $2 k+1$, then the bracket $[\mathbf{X}, \mathbf{X}]_{N}$ of this vector with itself is in general, not trivial. For example, let $\mathbf{X}=d x^{j} X_{j}^{i}(x) \partial_{i}$ be a vector field with values in usual 1 -forms on $M$, i.e. $\mathbf{X}=\mathbf{X}(x)$ is a section of the usual linear operators on the tangent bundle. The lifted vector field on $\Pi T M$ is equal to $\widehat{\mathbf{X}}(x, d x)=d x^{j} X_{j}^{i}(x) \partial_{i}-d x^{k} d x^{j} \partial_{k} X_{j}^{i}(x) \frac{\partial}{\partial d x^{i}}$. This is an odd vector field on $\Pi T M$. One can see that

$$
[\mathbf{X}(x), \mathbf{X}(x)]_{N}=2 d x^{j} d x^{r}\left(X_{j}^{m}(x) \partial_{m} X_{r}^{i}(x)+\partial_{r} X_{j}^{m}(x) X_{m}^{i}(x)\right) \partial_{i} .
$$

Now return to modular classes.

Let $\mathbf{X}(x, d x)$ be an arbitrary odd vector field on a manifold $M$ with values in differential forms, e.g. with values in 1-forms, and let $\boldsymbol{\eta}=\widehat{\mathbf{X}}(x, d x)$ be its lifting (34) to the supermanifold ПTM. According to Theorem 1, the pair $(\Pi T M, \boldsymbol{\eta})$ defines a supermanifold 
$\mathcal{N}=\Pi T M \times \Pi \mathbb{R}$ provided with an induced odd Poisson structure such that the modular class of this Poisson manifold is equal to

$$
\frac{1}{8}[\boldsymbol{\eta}, \boldsymbol{\eta}]=\frac{1}{8}[\widehat{\mathbf{X}}(x, d x), \widehat{\mathbf{X}}(x, d x)]=\frac{1}{8}\left[\widehat{\mathbf{X}, \mathbf{X}]_{N}} .\right.
$$

Given an arbitrary odd form-valued vector field $\mathbf{X}$ on a manifold we define an odd Poisson supermanifold such that its modular class is represented by Nijenhuis bracket $[\mathbf{X}, \mathbf{X}]_{N}$.

\section{Equation $\Delta^{2}=0$. Discussion}

Let $(M, \mathbf{E})$ be a supermanifold equipped with an odd Poisson bracket. What can we say about the solutions of the equation

$$
\Delta^{2}=0
$$

where $\Delta$ is a second order self-adjoint operator on half-densities with principal symbol $\mathbf{E}$ ? In other words, does there exist an odd potential $U(x)$ such that for the operator $\Delta=$ $\frac{1}{2}\left(E^{a b} \partial_{b} \partial_{a}+\partial_{b} E^{b a} \partial_{a}+U(x)\right)$, the condition $\Delta^{2}=0$ is obeyed? It follows from Proposition 4 that this equation has a solution if and only if the modular class of the odd Poisson manifold $(M, \mathbf{E})$ vanishes. Two operators $\Delta, \Delta^{\prime} \in \mathcal{F}_{\mathbf{E}}$ obey the equation $\Delta^{2}=\Delta^{\prime 2}=0$, where $\Delta^{\prime}=\Delta+F$, if and only if the Hamiltonian vector field $D_{F}$ vanishes, i.e. $F$ is a Casimir function of the Poisson bracket.

Equation (36) can be rewritten as the condition of the vanishing of the modular vector field $\mathbf{X}(\Delta): \Delta^{2}=\mathcal{L}_{\mathbf{X}}$. On setting the modular vector field (see (21)) equal to zero, we come to a system of differential equations in the potential $U$ :

$$
U(x): \mathbf{X}(\Delta)=0 \text {, i.e. },(-1)^{p(a)} E^{a b} \partial_{b} U+\frac{1}{2} \partial_{b}\left(E^{b c} \partial_{c} \partial_{p} E^{p a}\right)=0 .
$$

One can rephrase the statement above in the following way. These differential equations have a solution if and only if the modular class of the Poisson manifold vanishes, and these solutions are unique up to a Casimir function $F$ of the Poisson bracket. The modular class is the obstruction for a solution of this equation.

In the symplectic case (if the Poisson structure is non-degenerate,) the potential $U$ is defined up to an an odd constant (an auxiliary odd parameter). For the canonical odd Laplacian (26), this odd constant is equal to zero. In the absence of odd constants, equations (37) have unique solutions which are given by Bering's formula (27).

One can distinguish the following special case of an odd Poisson manifold when there exists a canonical odd Laplacian. Suppose that an odd Poisson manifold consists of odd symplectic leaves which are all of the same dimension $p \mid p$, (the tensor $\mathbf{E}$ defining the Poisson structure has constant rank $p \mid p)$, i.e. in a vicinity of an arbitrary point there are Darboux-Lie coordinates $\left(q^{i}, \theta_{j}, z^{\alpha}\right), i, j=1, \ldots, p\left(q^{i}\right.$ are even, $\theta_{j}$ are odd, $)$ such that

$$
\begin{gathered}
\left\{q^{i}, \theta_{j}\right\}=\delta_{j}^{i}, \quad\left\{q^{i}, q^{j}\right\}=0, \quad\left\{\theta_{i}, \theta_{j}\right\}=0, \quad(i, j=1, \ldots, k), \\
\left\{q^{i}, z^{\alpha}\right\}=\left\{\theta_{j}, z^{\alpha}\right\}=\left\{z^{\alpha}, z^{\beta}\right\}=0 .
\end{gathered}
$$

One can consider the following example of such a Poisson manifold: let $(M, \mathbf{P})$ be a usual Poisson manifold of constant rank, i.e. the Poisson tensor $\mathbf{P}$ has constant rank $2 k$ at all points of $M$. Then the supermanifold $\Pi T M$ with the Koszul bracket (28) consists of $p \mid p$ dimensional symplectic leaves $(p=2 k)$. 
In the case when there exists coordinates satisfying equations (38), one can define a canonical operator in a way similar to (26). In a vicinity of an arbitrary point, choose Darboux-Lie coordinates (38), and set $\Delta=\frac{\partial^{2}}{\partial q^{i} \partial \theta_{i}}$ in these local Darboux-Lie coordinates. As in equation (26), one can see that the operator is well-defined, it does not depend on the choice of Darboux-Lie coordinates. The potential of this operator vanishes in these coordinates. In the article [6] Bering wrote the formula for the canonical operator in the Poisson case which covers this example.

We must mention the following important fact: the canonical odd Laplacian assigns to an arbitrary volume form, the scalar function: $\boldsymbol{\rho} \mapsto \sigma_{\boldsymbol{\rho}}=\frac{\Delta \sqrt{\boldsymbol{\rho}}}{\sqrt{\boldsymbol{\rho}}}$. In the article [1], Batalin and Bering showed that this function is proportional to the scalar curvature of a torsion-free affine connection compatible with the symplectic structure and this volume form. In the paper [2] the authors generalised this result for a class of odd Poisson manifolds of constant rank (see (38)). The explanation of this statement will shed light on the geometry of the compensating field of the odd canonical Laplacian.

We will make one final remark.

It is a standard textbook fact that any Riemannian manifold $(M, \mathbf{G})$ possesses a unique symmetric connection compatible with the metric $\Gamma$ (the Levi-Civita connection). An explicit formula $\Gamma_{k m}^{i}=\frac{g^{i j}}{2}\left(\partial_{k} g_{m j}+\partial_{m} g_{k j}-\partial_{j} g_{k m}\right)$ expresses the Christoffel symbols of the Levi-Civita connection in terms of the functions $g_{i k}(x)$, which are the matrix entries of the Riemannian metric $\Gamma=g_{i k}(x) d x^{i} d x^{k}$. Any odd symplectic supermanifold possesses a unique odd potential $U(x)$ (it is a compensating field of the canonical odd Laplacian (26)), which vanishes in Darboux coordinates). Bering's formula (27) explicitly expresses this potential in terms of the functions $E^{i k}(x)$ which are the matrix entries of the non-degenerate Poisson tensor $\mathbf{E}=E^{a b} \partial_{b} \otimes \partial_{a}$ defining this odd symplectic structure.

For an affine connection compatible with the odd symplectic structure, the situation is very different. There are many symmetric affine connections compatible with a given symplectic structure on a manifold (one may take a connection such that its Christoffel symbols vanish in given set of Darboux coordinates). However there is no formula which expresses at least one of these connections in terms of the functions $\omega_{i k}(x)$, the matrix entries of the closed non-degenerate 2-form $\boldsymbol{\omega}=\omega_{i k} d x^{i} \wedge d x^{k}$ defining symplectic structure. Indeed, suppose that there exists an expression

$$
\Gamma_{k m}^{i}=\Gamma_{k m}^{i}\left(\omega_{p q}, \frac{\partial \omega_{p q}}{\partial x^{r}}, \ldots\right)
$$

such that if the functions $\omega_{p q}(x)$ are the components of a closed non-degenerate differential 2-form, then the left hand side of this equation defines the Christoffel symbols of a symmetric affine connection compatible with the symplectic structure defined by the 2 -form $\boldsymbol{\omega}=\omega_{i k} d x^{i} \wedge d x^{k}$. In a vicinity of an arbitrary point $x_{0}$, choose arbitrary Darboux coordinates $\left(x^{i}\right)$. In Darboux coordinates all the functions $w_{i k}(x)$ are constants. Hence the Christoffel symbols $\Gamma_{k m}^{i}$ are constants in arbitrary Darboux coordinates. This contradicts the existence of non-linear canonical transformations (transformations from Darboux coordinates to another Darboux coordinate system). The absence of an explicit formula comes from the non-uniqueness. 


\section{ACKNOWLEDGMENTS}

We are happy to acknowledge A.Voronov and B.Kruglikov for encouraging us to begin the work on this article. It is a pleasure to also thank K.Mackenzie and T.Voronov for very useful discussions. One of us (H.Kh.) learnt much about the peculiarities of Poisson geometry through discussions with A. Bolsinov. Many thanks to him.

\section{REFERENCES}

[1] Batalin, I.A. and Bering, K.: Odd scalar curvature in field-antifield formalism. J. Math. Phys., 49, 1-22, 033515, (2008).

[2] Batalin, I.A. and Bering, K.: Odd scalar curvature in anti-Poisson geometry. Phys. Lett. B., 663(1-2), 132-135, (2008).

[3] Batalin, I.A. and Vilkovisky, G.A.: Gauge algebra and quantization. Phys. Lett., 102B , 27-31, (1981).

[4] Berezin, F.A.: Introduction in superanalysis. (Expanded translation from the Russian: Introduction to analysis with anticommuting variables. Kirillov, A.A., (ed.), Moscow State Univerisity Press, Moscow (1983).) Translation edited by Leites, D.A. and Reidel, D., Dordrecht (1987).

[5] Bering, K.: A note on semidensities in antisymplecctic geometry. J. Math. Phys., 47, 1-9, 123513, (2006).

[6] Bering, K.: Semidensities, second-class constraints, and conversion in anti-Poisson geometry. J. Math. Phys., 49(4), 043516, 31, (2008).

[7] Hitchin, N.J., Segal, G.B. and Ward, R.S.: Integrable systems. The Clarendon Press. Oxford Univ, Univ.Press, New-York, (1999).

[8] Khudaverdian, O.M. (H.M.).: Geometry of Superspace with Even and Odd Brackets. J. Math. Phys. 32, 1934-1937, (1991). (Preprint of the Geneva University, UGVA-DPT 1989/05-613.)

[9] Khudaverdian, H.M.: Laplacians in odd symplectic geometry. In Quantization, Poisson Brackets and Beyond, ed. Theodore Voronov, Th., Contemp. Math., Vol. 315, pp. 199-212, Amer. Math. Soc., Providence, RI, 2002.

[10] Khudaverdian, H.M.: Semidensities on odd symplectic supermanifold. Comm. Math. Phys., 247, 353390, (2004). (Preprint 2000, arXive: math/0012256)

[11] Khudaverdian, H.M. and Voronov, Th.: On Odd Laplace operators. Lett. Math. Phys. 62, 127-142, (2002).

[12] Khudaverdian, H.M. and Voronov, Th.: On Odd Laplace operators II. Amer.Math.Soc.Transl.(2) 212, 179 (2004).

[13] Khudaverdian, H.M. and Voronov, Th.: Geometry of differential operators of second order, the algebra of densities, and groupoids. J. Geom. Phys. 64, 31, (2013). (See also preprint of Max-Planck-Institut for Math., 73, (2011).)

[14] Khudaverdian, H.M. and Voronov, Th.: Geometric constructions on algebra of densities. In Topology, Geometry, Integrable Systems, and Mathematical Physics: Novikov's Seminar 2012-2014 eds. Buchstaber, V. M., Dubrovin, B. A., and Krichever, I. M., AMS Translations, Ser. 2, 234, 221-243, Providence, RI, 2014, math-arXiv:1310.0784.

[15] Kosmann-Schwarzbach, Y. and Monterde, J.: Divergence operators and odd Poisson brackets. Ann. Inst. Fourier (Grenoble) 52,(2),419-456, 2002.

[16] Ovsienko, V. and Tabachnikov, S.: Projective Differential Geometry Old and New. From Schwarzian Derivative to the Cohomology of Diffeomorphism Groups. (Cambridge University Press, 2005).

[17] Schwarz, A.S.: Geometry of Batalin-Vilkovisky Formalism. Commun. Math. Phys., 155, 249-260, (1993).

[18] Weinstein, A: The local structure of Poisson manifolds. J. Diff. Geom., 18(3), 523-557, (1983)

[19] Weinstein, A.: The modular automorphism group of a Poisson manifold. J. Geom. Phys., 23(3-4), 379-394, 1997. 
School of Mathematics, University of Manchester, Oxford Road, Manchester M13 9PL, $\mathrm{UK}$

E-mail address: khudian@manchester.ac.uk, matthew.peddie@manchester.ac.uk 